\title{
O Uso de Escalas Psicológicas para Avaliar Autoestima
}

\author{
Daniela Bergesch D’Incao Marrone ${ }^{1}$, Luciana Karine de Souza, Claudio Simon Hutz \\ Universidade Federal do Rio Grande do Sul, Porto Alegre-RS, Brasil
}

\section{RESUMO}

A autoestima é amplamente explorada em pesquisa. Dois estudos foram conduzidos para identificar o uso das escalas de autoestima em publicações científicas. O primeiro analisou artigos científicos publicados em quatro periódicos tradicionais no campo da avaliação psicológica no Brasil. A escala mais utilizada foi a Escala de Autoestima de Rosenberg (RSES), referida em 68\% dos casos. Além disso, houve predomínio da avaliação global da autoestima. O segundo estudo avaliou publicações disponíveis no Portal de Periódicos CAPES/ MEC. Notou-se um aumento de publicações com escalas de autoestima a partir da década de 1990; a RSES foi novamente a mais citada (64\%). Atenta-se para a importância de explorar diferentes instrumentos de avaliação, contemplando diferentes domínios e situações que influenciam diretamente os níveis de autoestima. Acima de tudo, detectou-se uma notável ausência de informação sobre as propriedades psicométricas das escalas utilizadas.

Palavras-chave: escala; autoestima; avaliação.

\section{ABSTRACT - The Use of Psychological Scales to Assess Self-Esteem}

Self-esteem has been widely investigated in research. Two studies were performed to identify the use of self-esteem scales in scientific publications. The first study analyzed papers published in four traditional journals in the field of psychological assessment in Brazil. The most used scale was the Rosenberg Self-Esteem Scale (RSES), cited by $68 \%$ of the papers. In addition, assessment of global self-esteem predominated. The second study assessed publications available in the Portal de Periódicos CAPES/MEC database. There was an increase in publications citing self-esteem scales during the 1990's; the RSES was again the most cited (64\%). We highlight the relevance of exploring different assessment instruments, including different domains and situations that directly influence self-esteem levels. More importantly, we detected a remarkable absence of information on the psychometric properties of the scales used.

Keywords: scale; self-esteem; assessment.

\section{RESUMEN - El Uso de Escalas Psicológicas para Evaluar Autoestima}

La autoestima es ampliamente estudiada en investigación científica. Se realizaron dos estudios para identificar el uso de las escalas de autoestima en publicaciones científicas. El primero analizó artículos científicos publicados en cuatro periódicos tradicionales en el campo de la evaluación psicológica en Brasil. La escala más utilizada fue la Escala de Autoestima de Rosenberg (RSES), empleada por $68 \%$ dos casos. Además, prevaleció la evaluación global de autoestima. El segundo estudio evaluó publicaciones disponibles en el Portal de Periódicos CAPES/MEC. Se identificó un aumento de publicaciones con escalas de autoestima a partir de la década de 1990; la RSES fue nuevamente la más citada (64\%). Se enfatiza la importancia de explorar diferentes instrumentos de evaluación, contemplando diferentes dominios y situaciones que influyen directamente en los niveles de autoestima. Sobre todo, se detectó una notable ausencia de información sobre las propiedades psicométricas de las escalas utilizadas.

Palabras-clave: escala; autoestima; evaluación.

O aumento da autoestima é um argumento recorrente em publicações de diversas áreas do conhecimento, nos resultados de relatos de pesquisas ou de intervenções. Construto fundamental em Psicologia, diz respeito à avaliação que a pessoa faz sobre si mesma, mais especificamente, sobre a avaliação do conceito de si - seu autoconceito. Todavia, nem autoestima, nem autoconceito possuem definição única em Psicologia, gerando consequências importantes sobre a escolha do instrumento para avaliar como o sujeito se valoriza.

Cunhado por William James (1890/1950), a autoestima foi assunto do primeiro livro de Psicologia publicado nos Estados Unidos, consolidando-se como um dos mais antigos temas estudados na área. Considerandose o constante e crescente número de publicações, percebe-se que ainda há muito interesse no assunto (Mruk, 2013). 
Apesar disso, não há uma definição unânime do construto. Atualmente há três formas de compreendê-la: Autoestima Global (traço), Sentimentos de Autovalor (estado) e Autoavaliação (autoestima por domínios específicos). Autoestima Global envolve como um indivíduo geralmente se sente com relação a si mesmo. A autoestima enquanto sentimentos de autovalor abrange a avaliação de eventos de vida que ajudam a elevá-la ou reduzi-la. A forma de autoavaliação por domínios considera que cada indivíduo possui diferentes níveis de autoestima em distintos domínios da vida (por exemplo, trabalho, saúde etc.) (Brown \& Marshall, 2006; Harter, 1986).

Para avaliação da autoestima, a escala mais amplamente usada e considerada padrão ouro é a Escala de Autoestima de Rosenberg (Rosenberg Self-Esteem Scale RSES) (Hutz \& Zanon, 2011; Mruk, 2013). Trata-se de uma medida que avalia a autoestima global, é unidimensional e formada por dez afirmações relativas a sentimentos de autoestima e autovalia. Os itens são respondidos por meio de uma escala tipo Likert de quatro pontos, com 1 correspondente a discordo totalmente; 2, a discordo; 3, a concordo; e 4, a concordo totalmente (Hutz \& Zanon, 2011). Essa escala foi adaptada para o português brasileiro por Hutz (2000) e revisada por Hutz e Zanon (2011), indicando adequadas propriedades psicométricas. A escala está totalmente disponível em Hutz, Zanon, e Vazquez (2014), juntamente com suas normas, forma de aplicação e interpretação dos resultados por sexo e faixa etária.

Para um panorama recente do amplo uso da RSES em trabalhos acadêmicos, uma busca simples na plataforma Google Acadêmico pela expressão "escala de autoestima de Rosenberg" (incluindo as aspas na busca) gerou 2360 resultados de citações. Não há dúvidas, portanto, da profunda inserção dessa medida em distintas áreas do conhecimento.

A autoestima congrega aspectos fisiológicos, neurológicos, bem como aspectos psicológicos mais universais, como as emoções e a avaliação que a pessoa faz sobre si, sobre o mundo e sobre seu futuro. Em outras palavras, reúne aspectos cognitivos e afetivos. No entanto, não são menos importantes os aspectos culturais, construídos socialmente e fundamentados em significados compartilhados e expectativas sociais. Assim, a autoestima de fato precisa ser aprendida dentro do grupo cultural, onde ela é vivenciada e, portanto, sujeita ao controle social e a circunstâncias específicas que a evocam (Hewitt, 2009). Esses aspectos sinalizam a necessidade da atualização das medidas em autoestima, em qualquer das abordagens adotadas.

Portanto, a definição de autoestima global já não é suficiente para explicar o construto. Ademais, perspectivas mais contemporâneas passaram a considerar a autoestima não só como um traço, mas também como um estado. Consequentemente, deixa-se de pensar em um contínuo com extremos de baixa ou alta autoestima, para se pensar em aspectos interligados e dependentes de situações distintas (Mruk, 2013).
Por que é importante avaliar a autoestima? Porque ela repercute em uma ampla gama de comportamentos que se estende por todo o ciclo vital (Mruk, 2013). Há um amplo corpo de evidências que mostram associações entre baixa autoestima e humor negativo, percepção de incapacidade, delinquência, depressão, ansiedade social, transtornos alimentares e ideação suicida. Também há maciças provas científicas de associação entre elevada autoestima e saúde mental, habilidades sociais e bem-estar (Hutz, Midgett, Pacico, Bastianello, \& Zanon, 2014; Hutz et al., 2014).

Outro problema com o uso da autoestima é como sinônimo de satisfação com a participação em intervenções. A verbalização de aumento de satisfação com a participação ou envolvimento em qualquer intervenção que seja - educativa, esportiva, recreativa, social etc. - não equivale à elevação da autoestima. Esta, não tendo sido avaliada no programa ou projeto, não pode ser simplesmente inserida na equação e utilizada como argumento. No máximo, elevou-se a autoestima do pesquisador ou educador que realizou a intervenção. Ademais, esse argumento equivale a tratar a autoestima com base em um entendimento de senso comum, desconsiderando-a um construto psicológico cientificamente fundamentado.

Há construtos controversos em Psicologia e que vêm desafiando pesquisadores que buscam avaliá-los. Um exemplo atual é a resiliência, cuja definição é complexa e requer atenção a elementos contextuais, desenvolvimentais e interacionais que a constituem. $\mathrm{O}$ trabalho de Reppold, Mayer, Almeida, e Hutz (2012) examinou o uso de escalas para avaliar resiliência e, dentre as conclusões traçadas, apontou que "os itens das escalas consideram fatores de risco e de proteção que não podem ser generalizados às diversas faixas etárias, quadros socioeconômicos ou grupos culturais" (p. 253). Reppold et al. (2012) mostram que estudos sobre o uso de escalas, que a princípio informam sobre a situação de um determinado instrumento, contribuem para alertar para a necessidade de constante atualização quanto a instrumentos psicológicos (seja para pesquisa, seja para aplicação profissional) e de congruência entre construto e instrumento.

O presente estudo surgiu a partir das reflexões recém-apresentadas. Em primeiro lugar, da presença frequente de escalas de autoestima em publicações em Psicologia, sinalizando para um uso despreocupado em termos de atualização teórica e consistência psicométrica. Em segundo, pela argumentação de aumento de autoestima sem a devida medida dessa alteração. Em terceiro lugar, pelo desenvolvimento teórico na área, que vem demonstrando um movimento em direção a um construto mais contextualizado, afinado a culturas, etapas do desenvolvimento humano e situações específicas.

Como objetivo principal, este trabalho buscou averiguar o uso de escalas psicológicas para avaliar a autoestima em publicações especializadas. Como objetivos específicos, buscou-se identificar as escalas, suas 
características psicométricas e evidências de validade. Espera-se contribuir para que o uso da avaliação da autoestima em trabalhos brasileiros envolva instrumentos confiáveis e cientificamente fundamentados.

Dois estudos são relatados. No primeiro, quatro revistas científicas nacionais em Psicologia são fonte de dados para o Estudo 1. No segundo, foram consultados artigos científicos publicados no Portal de Periódicos CAPES/MEC.

\section{Estudo 1: O Uso de Escalas para Avaliar Autoestima em Publicações Nacionais em Psicologia}

Nesse primeiro estudo, buscou-se conhecer o uso de escalas para avaliar autoestima em publicações nacionais indexadas em Psicologia. Primeiramente, é descrito o método adotado, seguido dos resultados e da discussão.

\section{Método}

No meio científico psicológico brasileiro, há periódicos tradicionais que vêm mantendo estável liderança e qualidade em termos de publicações científicas. Também por serem das revistas mais antigas em funcionamento, optou-se pela inclusão dos periódicos Psicologia: Reflexão e Crítica e Psicologia: Teoria e Pesquisa no conjunto de revistas a serem consultadas para a averiguação do uso de escalas de autoestima no meio acadêmico em Psicologia no país. Foram incluídas as revistas Avaliação Psicológica, por sua especialidade, e Psico-USF, por tradicionalmente ser veículo de publicações em avaliação psicológica em virtude de seu Programa de Pós-Graduação em Psicologia focalizar sobremaneira nessa área. Escolhidos os periódicos-alvo, procedeu-se à busca pelas publicações.

Os acervos foram consultados a partir da home page das revistas indexadas nas bases de dados SciELO (www.scielo.org) e PePSIC (pepsic.bvsalud.org). O acervo da revista Avaliação Psicológica está disponibilizado na íntegra; a revista Psicologia: Reflexão e Crítica disponibiliza desde o volume 10 (1997); a Psico-USF conta a partir do volume 6 (2001); e a revista Psicologia: Teoria e Pesquisa dispõe a partir do volume 15 (1999). Os volumes indisponíveis na Internet foram consultados na forma impressa, para que todo o acervo de cada periódico fosse analisado.

A busca considerou os artigos nos quais constassem os termos "escala" e "autoestima" ou "auto-estima" (grafia modificada em 2009, com a retirada do hífen). O campo de busca foi preenchido da seguinte forma: escala autoestima. A coleta dos textos foi realizada em 20/02/2018 nos quatro periódicos selecionados.

\section{Resultados}

As buscas geraram as seguintes frequências de publicações com uso de escalas sobre autoestima: 1. Avaliação Psicológica: 10 artigos; 2. Psicologia: Reflexão e Crítica: 11; 3. Psicologia: Teoria e Pesquisa: seis; e 4. Psico-USF: seis publicações. Os 33 textos foram lidos na íntegra com o objetivo de identificar os dados detalhados sobre o instrumento utilizado para avaliar autoestima no trabalho. Nesse processo de leitura, foram identificadas duas revisões de literatura (sem coleta de dados) as quais foram retiradas da amostra. A amostra final contou de 31 artigos.

A Tabela 1 mostra especificamente aquelas publicações que utilizaram a RSES nos quatro periódicos examinados, totalizando 16 casos. São detalhados o ano da publicação do artigo, a fonte citada pelos autores como origem da RSES, a amostra utilizada, a citação da versão adaptada e validada, e o valor do coeficiente de fidedignidade obtido com a amostra (alfa de Cronbach) ou citado na seção de instrumentos.

Tabela 1

Uso da Escala de Autoestima de Rosenberg em Quatro Periódicos Nacionais de Psicologia

\begin{tabular}{ccccc}
\hline $\begin{array}{c}\text { Ano do } \\
\text { artigo }\end{array}$ & Fonte citada no artigo & Amostra & Adaptação e validação & $\begin{array}{c}\text { alfa de } \\
\text { Cronbach }\end{array}$ \\
\hline 1995 & N.I. & BR & N.I. & N.I. \\
2010 & Rosenberg (1965) & BR & Hutz (2000) & 0,70 \\
2010 & Rosenberg (1986) & BR & Assis et al. (2003) & 0,68 \\
2011 & Rosenberg (1979) & BR & Hutz \& Zanon (2011) & 0,90 \\
2011 & Rosenberg (1989) & BR & Hutz \& Zanon (2011) & 0,90 \\
2012 & Rosenberg (1989) & BR & Hutz (2000) & 0,80 \\
2012 & Rosenberg (1965) & BR & Hutz \& Zanon $(2011)$ & 0,90 \\
2013 & Rosenberg (1989) & BR & Hutz \& Zanon $(2011)$ & 0,90 \\
2013 & Rosenberg (1989) & BR & Hutz \& Zanon $(2011)$ & 0,81 \\
2013 & HR & Hutz \& Zanon $(2011)$ & N.I. \\
2014 & Rosenberg (1965) & BR & Hutz \& Zanon $(2011)$ & 0,85 \\
2015 & Rosenberg (1985) & BR & Dini, Quaresma, \& Ferreira $(2004)$ & Dini et al. (2004) \\
2015 & Dini et al. (2004) & BR & & 0,64 \\
\hline
\end{tabular}


Tabela 1 (continuação)

Uso da Escala de Autoestima de Rosenberg em Quatro Periódicos Nacionais de Psicologia

\begin{tabular}{ccccc}
\hline $\begin{array}{c}\text { Ano do } \\
\text { artigo }\end{array}$ & Fonte citada no artigo & Amostra & Adaptação e validação & $\begin{array}{c}\text { alfa de } \\
\text { Cronbach }\end{array}$ \\
\hline 2015 & Rosenberg $(1965,1979)$ & Espanha & NI & NI \\
2016 & Rosenberg (1989) & BR & Hutz \& Zanon (2011) & 0,93 \\
2017 & Dini et al. (2004) & BR & Dini et al. (2004) & 0,87 \\
\hline
\end{tabular}

Nota. RSES=Rosenberg Self-Esteem Scale; NI=não informado; BR=Brasil

O tratamento dado por Hutz (2000) e por Hutz e Zanon (2011) à RSES foi citado em 12 dos 16 casos, demonstrando serem estes autores os mais referidos na adaptação cultural e fornecimento de adequadas evidências de validade para a escala ser utilizada com amostras brasileiras. $\mathrm{O}$ índice de confiabilidade indicado nas publicações variou de 0,64 a 0,93 , incluído os índices encontrados por Hutz $(0,90)$ e por Hutz e Zanon $(0,90)$. As publicações datam dos anos entre 1995 e 2017.

Citado três vezes, o artigo de Dini, Quaresma, e Ferreira (2004) foi o segundo mais frequente nos trabalhos encontrados. Com base em Rosenberg (1989), os autores realizaram tradução, retrotradução, discussão em grupo multidisciplinar de saúde, análise de equivalência cultural e estudos de evidências de validade de face, de construto e de conteúdo. Cabe destacar que o contexto do trabalho - cirurgia plástica - contou com a participação de pacientes inscritos para a realização de cirurgia em ambulatório de um hospital universitário. Ainda que se trate de um trabalho detalhado, já soma 14 anos de publicação. Esse aspecto é importante em virtude das mudanças nos valores de uma sociedade, no comportamento dos indivíduos, bem como novas tendências e desafios que podem alterar o modo como as pessoas valorizam a si e a suas vidas. Assim como Dini et al. (2004), Avanci, Assis, Santos, e Oliveira (2007) conduziram sua própria adaptação da RSES, citada em dois artigos.

O trabalho de Assis et al. (2003), citado uma vez, também já considerado antigo, obteve um coeficiente de confiabilidade relativamente baixo $(0,68)$, o que não favorece a versão dos autores para uso na avaliação da autoestima. Os autores realizaram sua própria adaptação cultural da escala, com tradução e retrotradução, análise por expert linguístico, discussão com grupo de experts no tema, avaliação psicométrica e piloto com população-alvo.

A Escala de Autopercepção de Harter (1985) foi mencionada em duas publicações. Esse instrumento segue a abordagem multidimensional da avaliação das percepções de si nos domínios cognitivo, físico, social e comportamental, bem como mede o senso de autovalor global da criança. A escala original continha quatro domínios, ampliados para seis na versão de 1985: competência acadêmica, aceitação social, competência atlética, aparência física, comportamento e autoestima global. É essa versão de 1985 a citada nos dois trabalhos encontrados.
Atualmente o instrumento é chamado de Self-Perception Profile e está disponível nos formatos para crianças de oito a 13 anos, adolescentes de 14 a 19 anos, adultos emergentes, estudantes universitários, adultos de 20 a 60 anos, idosos e estudantes com dificuldades de aprendizagem de oito a 18 anos (cf. https://portfolio.du.edu/SusanHarter/ page/44210).

Outro instrumento de autoria de Susan Harter foi a Escala de Competência Percebida (Harter, 1982), que possui uma subescala de autoestima geral. Os autores, no entanto, não informaram o alfa de Cronbach, como a versão em língua portuguesa foi elaborada, nem apresentaram evidências de validade da versão utilizada (Pasin, Paiva, \& Lannes, 2012).

Os demais dois trabalhos localizados na busca realizada foram citados uma vez, cada qual com características distintas das anteriormente descritas. A Escala de Cognições Acadêmicas Autorreferentes é composta por diferentes fatores, um dos quais avalia a autoestima escolar, com índice de fidedignidade de 0,72 (Costa, Gomes, \& Fleith, 2017). O outro estudo utilizou a Escala Multidimensional de Autoestima criada por Coopersmith (1967, 1989), e refere apenas que foi realizada uma tradução da escala original com 58 itens, incluindo aqueles de desejabilidade social (Gobitta \& Guzzo, 2002). A data da coleta de dados e a escassez de informações psicométricas sobre a versão realizada desfavorecem a escolha pelo instrumento referido (Capelatto, Lima, Ciasca, \& SalgadoAzoni, 2014).

Um dos artigos indicou o uso de uma escala que avalia a valência negativa da autoestima. Carneiro e Baptista (2016) utilizaram em seu estudo a Escala de Pensamentos Depressivos. Esta foi elaborada com base na proposta de tríade cognitiva de Beck e avalia pensamentos distorcidos que podem indicar depressão. Divide-se em dois fatores: o primeiro avalia baixa autoestima e desesperança por meio de 16 itens; o alfa desse fator foi 0,93 .

Encontrou-se um estudo que buscou evidências de validade e fidedignidade em amostras brasileiras da Escala de Avaliações Autorreferentes (Judge, Erez, Bono, \& Thoresen, 2003). Essa escala avalia quatro traços que definem avaliações autorreferentes por meio de um instrumento unifatorial. Foi realizada tradução para o português, retrotradução para inglês e, a partir de uma comparação de ambas, foi ajustada a versão final em português. O alfa de Cronbach obtido foi de 0,78. Outro 
estudo que vai nessa mesma linha investigou as propriedades psicométricas da Escala Multidimensional de Autoconceito. Nesse foi utilizada uma versão adaptada da Escala de Autoconceito Forma 5 (García \& Musitu, 2014), contando com apenas quatro dos cinco fatores da escala original. Essa opção foi justificada com vistas a reduzir o volume de itens a responder, já que havia outro instrumento que avaliou o aspecto emocional. Os índices de confiabilidade foram 0,86 (componente acadêmico), 0,76 (componente familiar), 0,66 (componente social) e 0,76 (componente físico).

Por fim, outro estudo realizou a tradução e adaptação do Inventário de Coping Parental para avaliar a autoestima. Para tanto, foi realizada uma tradução do inventário para o português por dois tradutores bilíngues e comparadas as duas versões para atestar a acurácia da versão brasileira. $\mathrm{O}$ inventário constitui-se de três fatores: um avalia a autoestima parental levando em conta os construtos de autoeficácia, estabilidade psicológica e apoio social. $\mathrm{O}$ alfa não foi informado.

\section{Discussão}

Os resultados não chegam a mostrar um quadro preocupante com relação às escolhas por escalas para avaliar a autoestima em estudos nacionais publicados em quatro periódicos científicos de referência na área da Psicologia e da instrumentação psicológica. Todavia há razão para algum alarme, como será discutido a seguir.

De fato, os estudos que trazem evidências sólidas de validade para a RSES - a mais citada e utilizada no meio acadêmico - são os trabalhos de Hutz e Zanon (2011) e de Hutz et al. (2014), ambos precedidos por Hutz (2000). Com amostras brasileiras, essa versão tem obtido resultados consistentes da correlação positiva entre a autoestima e, por exemplo, afetos positivos $(0,65)$, satisfação de vida $(0,47)$, esperança $(0,60)$, otimismo $(0,60)$ (cf., Zanon, Bastianello, Pacico, \& Hutz, 2013), autocompaixão $(0,47)$ e autoeficácia $(0,50)$ (ambas em Souza \& Hutz, 2016).

As demais versões, no entanto, necessitam de revisão com novas amostras, com informações psicométricas explicitadas e boas evidências de validade. De todo modo, o trabalho mais citado e disponível se mostra suficientemente adequado para ser utilizado no Brasil, e está totalmente à disposição sem necessidade de requisição especial aos autores (Hutz et al., 2014).

Evidencia-se o uso predominante da avaliação da autoestima de forma global nos estudos contemplados nos periódicos brasileiros. Atualmente, apesar dessa modalidade ainda ser muito utilizada na literatura internacional, há uma tendência para tratar esse construto por meio de diferentes domínios. Não é uma tendência exclusiva à autoestima, mas nas pesquisas em geral. Exemplo disso é a discussão acerca da inteligência múltipla e do quociente de inteligência (Marsh, Craven, \& Martin, 2013).
As pessoas privilegiam certos aspectos de suas vidas, sobre os quais depositam maiores expectativas. Como consequência, esses domínios mostram-se fundamentais para a manutenção da autoestima. Para alguns, o maior valor repousa sobre a carreira; para outros, a forma física, e assim por diante. Dessa forma, cada domínio pode ser ameaçado diante de falhas ou rendimento aquém da expectativa, gerando respostas negativas, isto é, avaliações negativas sobre si (Crocker \& Wolve, 2001).

Um contexto em que a informação sobre autoestima poderia ser mais bem aproveitada é diante de situações de cirurgia. Mais especificamente, a avaliação da autoestima no domínio da cirurgia plástica pode contribuir mais para a questão do que uma avaliação de autoestima global. Tal foi o caso em Dini et al. (2004), com amostra de pacientes prontos à cirurgia plástica. Possivelmente a avaliação da autoestima em domínios como o corpo, a face, a autoimagem, colaboraria com informações mais precisas com respeito às mudanças advindas da cirurgia. Ademais, é muito provável que o escore de autoestima do sujeito aumente após uma cirurgia plástica, por exemplo, para corrigir um defeito congênito ou oriundo de trauma. Espera-se o mesmo aumento em casos de busca por melhoria no domínio da estética corporal.

Conforme mostrou o Estudo 1, a RSES foi o instrumento mais utilizado na avaliação da autoestima, presente em 16 dos 31 casos. Em mais da metade dos estudos que utilizaram a RSES, foi referida a versão de Hutz (2000) e/ou de Hutz e Zanon (2011). Ainda assim, há relatos de índices baixos de confiabilidade nesses trabalhos, bem como outros problemas nas publicações. Estão ausentes dados psicométricos importantes sobre as adaptações realizadas sobre os instrumentos. A questão da idade das versões também se destaca, o que merece maior atenção por parte dos pesquisadores, especialmente aqueles que não estão familiarizados com o uso de instrumentos para avaliar construtos psicológicos. A seguir, é apresentado o Estudo 2.

\section{Estudo 2: O Uso de Escalas para Avaliar Autoestima em Publicações do Portal de Periódicos CAPES/MEC}

O Estudo 2 buscou localizar as escalas para avaliar autoestima utilizadas em pesquisas publicadas em periódicos científicos disponíveis no Portal de Periódicos CAPES/MEC. Inicia-se pela descrição do método, seguido dos resultados e discussão.

\section{Método}

Em 06/12/2017 foi realizada uma busca por resumos de publicações disponíveis no Portal de Periódicos CAPES/MEC. Optou-se por esse portal em virtude da grande quantidade de bases de dados bibliográficas que abriga. No entanto, foram determinados critérios de 
busca focalizados, dado o amplo uso de escalas de autoestima na produção científica de diferentes áreas do conhecimento, como referido na introdução do presente trabalho. Dessa forma, a busca de tipo avançada foi a opção para a investigação das publicações no portal.

Em busca avançada, no primeiro campo foi selecionada a opção no título, no segundo manteve-se a opção default contém, e no terceiro inseriu-se a expressão de busca self-esteem scale. Os demais campos e opções de critérios não foram acionados. Obtida a lista de resumos, clicou-se no critério de periódicos revisados por pares, por serem estes considerados mais criteriosos na avaliação de suas publicações. Restaram 146 resumos.

Os 146 resumos foram checados para repetições e para resumos de publicações que não fossem artigos científicos. Foram excluídas todas as repetições, resumos de trabalhos apresentados em congressos e resumos que não utilizaram coleta de dados com escala de autoestima. Um resumo foi excluído por estar redigido somente em japonês. Ao final, restaram 103 resumos para análise.

\section{Resultados}

Os 103 artigos foram publicados entre 1971 e dezembro de 2017, sendo somente um artigo de 1971 e os demais todos com data de publicação de 1990 em diante. O ano mais frequente de publicação foi o de 2016, com dez artigos. Chama a atenção o aumento nas publicações que utilizaram alguma escala de autoestima: na década de 1991 a 2000, foram 16 artigos; de 2001 a 2010, foram 40 artigos; e de 2011 até dezembro de 2017, 46 trabalhos publicados.

De fato, a escala mais citada e utilizada nos trabalhos foi a RSES. A escala foi encontrada em 66 publicações (64\% da amostra). Além disso, dois trabalhos referiram a Rosenberg's Stability of Self Scale. Foi identificada também a Modified State Rosenberg Self-Esteem Scale, em um estudo, e o uso único da State Self-Esteem Scale, a qual segundo os autores foi baseada na RSES.

Excluindo-se as escalas criadas por Rosenberg, foram identificadas 31 escalas diferentes para avaliar autoestima. O instrumento mais utilizado após a RSES foi a State Self-Esteem Scale, encontrada em quatro estudos.

Seis escalas foram criadas para avaliar a autoestima em contextos específicos, a saber: Self Esteem Scale for the Played Instrument, Organization-Based Self-Esteem Scale, Physical Disability Sexual and Body Esteem Scale, Body Weight, Image and Self-Esteem Evaluation Questionnaire, God-Centered Self-Esteem Scale, e Religious Collective SelfEsteem Scale. Outras seis escalas de autoestima dedicaram-se a avaliá-la especificamente em crianças, adolescentes ou estudantes universitários. Três trabalhos utilizaram a Collective Self-Esteem Scale e dois usaram a Relational Self-Esteem Scale.

Os 66 estudos encontrados que utilizaram a RSES foram analisados separadamente quanto às suas características principais. Quando o resumo não fornecia dados suficientes, o artigo completo era consultado.

Três grupos foram criados para organizar os 66 resumos que trataram da RSES. No primeiro, Grupo I $(n=44)$, os trabalhos dedicaram-se a analisar a escala em outro idioma ou cultura, destacando propriedades psicométricas, tradução e validação e/ou diferenças quanto a grupos culturais. A escala foi utilizada nos idiomas inglês na maioria dos artigos, bem como em mandarim (sete artigos), português (cinco), francês (cinco), espanhol (três), japonês (três), alemão (três), estoniano, grego, holandês, italiano, polonês, romeno, sérvio, sueco, tailandês, urdu e a língua de sinais norte-americana. Além disso, dois estudos envolveram mais de um país, um dos quais referiu que utilizou a RSES em 28 idiomas distintos. Quanto aos países envolvidos, foi possível identificar 28: África do Sul, Alemanha, Brasil, Canadá, Chile, China, Colômbia, Espanha, Estados Unidos, Estônia, França, Grécia, Holanda, Itália, Japão, Nova Zelândia, Paquistão, Polônia, Portugal, Quênia, Reino Unido, Romênia, Sérvia, Singapura, Suécia, Suíça, Tailândia e Taiwan. Cabe notar que dos 28 países pertencentes à União Europeia, 13 constam na listagem. Países asiáticos foram mais frequentes, na sequência, com sete casos.

Em complemento à tradicional busca pelo aperfeiçoamento das propriedades psicométricas das escalas em outro idioma (afora o inglês) e/ou grupo étnico-cultural, os estudos do Grupo I focalizaram elementos como a estrutura bifatorial da escala (dimensão negativa e dimensão positiva), o conteúdo de seus itens (wording), bem como seu desempenho conforme a teoria de resposta ao item. Poucos estudos envolveram outras variáveis não relacionadas à avaliação de si; no caso, três artigos incluíram medidas de personalidade e um trabalho verificou a autoestima em pacientes com doença coronariana, buscando adaptar a escala para esta condição de saúde.

No Grupo II de artigos que utilizaram a RSES $(n=15)$, há estudos psicométricos da escala em seu idioma original, com análise de suas dimensões e estrutura fatorial, análise de fidedignidade, análise pela teoria de resposta ao item, estudos de validade de critério, de conteúdo, de construto, análise da escrita de itens (wording) e a proposta de uma versão computadorizada. O Grupo III $(n=7)$ foi composto pelos artigos que utilizaram a RSES de modo secundário: cinco deles para estudo de validade de critério de outra medida de autoestima, e um para correlacionar com outras medidas de bem-estar psicológico.

\section{Discussão}

Além da RSES, foram identificadas 31 escalas para avaliar autoestima. Em sua maioria pertencem à abordagem global da autoestima, logo seguidas pela abordagem do estado e sentimento de autovalor. Encontrouse, também, a abordagem a domínios específicos que chamam a atenção pela diversidade de temáticas, como 
espiritualidade, desempenho musical com instrumento e condições de saúde e percepção corporal.

A autoestima passou por altos e baixos. Nos Estados Unidos durante a década de 1980, mais especificamente na Califórnia, foi dado um grande incentivo a estudos e técnicas que aumentassem a autoestima - movimento chamado de Self-Esteem Movement. Entretanto, em meados da década seguinte o construto passou a gerar dúvidas e a receber duras críticas. Dentre elas destaca-se a de Seligman (1995), que apontou para a possibilidade de, na busca incessante pela autoestima, pais e professores poderiam estar tornando uma geração de indivíduos mais vulneráveis à depressão. Outros pesquisadores ainda sugeriram a associação de níveis elevados de autoestima com alguns comportamentos inadequados como, por exemplo, violência, egotismo e narcisismo (Baumeister, Smart, \& Boden, 1996).

Nos primórdios da Psicologia Positiva, fundada por Seligman (1995) no final dos anos 90, a autoestima pouco se inseriu nos temas explorados por essa nova perspectiva. No primeiro manual de Psicologia Positiva (Snyder \& Lopez, 2002) pouca atenção foi dada ao construto, tratando-o mais como reflexo do mundo ocidental do que como um construto importante. Em um segundo momento, pesquisadores da Psicologia Positiva passaram a incluir o tema autoestima em diversas de suas pesquisas (Mruk, 2013).

Tendo isso em mente, destaca-se o notável aumento no uso de escalas de autoestima a partir de 2001, como mostram os resultados do Estudo 2. Uma possível razão para tal tenha sido o surgimento do movimento da Psicologia Positiva, em 1999, com a publicação seminal de Martin Seligman na revista American Psychologist. O psicólogo norte-americano convocou psicólogos e cientistas em Psicologia a direcionarem esforços para estudos e pesquisas sobre aspectos positivos da experiência humana. Nesse contexto, tanto construtos como esperança e otimismo passaram a receber maior atenção científica, como novos trabalhos foram desenvolvidos para retomar e, possivelmente, renovar a linha de pesquisa em autoestima. No Brasil, isso é claramente notado quando Hutz (2000) oferece a primeira adaptação da RSES, em 2011 revisa suas propriedades psicométricas (Hutz \& Zanon, 2011) e em 2014 apresenta novos estudos com normas para interpretação por sexo e faixa etária, bem como separadas em amostras de estudantes universitários e de adultos não estudantes (Hutz et al., 2014).

Nessa mesma direção, percebe-se a amplitude de estudos que utilizaram a RSES em versão com outros idiomas. Foram identificados 16 idiomas diferentes, inglês à parte, assim como a língua norte-americana de sinais. Essa expansão em idiomas no uso da RSES pode igualmente estar atribuída à renovação do interesse na investigação da autoestima, instigada pelo movimento da Psicologia Positiva, como argumentado anteriormente. Ademais, uma maior comunicação entre cientistas de diferentes países, facilitada pelo crescimento tecnológico atrelado a contextos como redes sociais virtuais, crescente acesso aberto a revistas científicas e diversidade de ferramentas de comunicação via Internet, também podem ter contribuído para o aumento de estudos de adaptação e validação cultural da RSES.

A investigação da autoestima por domínios não é nova (Harter, 1986). Há pesquisas focalizando domínios como o social, o atlético e o acadêmico. Nessa direção, von Soest, Wichstrom, e Kvalem (2016) examinaram o desenvolvimento da autoestima em 3116 noruegueses, de ambos os sexos, com idades entre 13 e 31 anos, tanto na forma global, como por domínios. Com delineamento longitudinal e sequencial por coortes, os autores abordaram a autoestima global, social, acadêmica, atlética e relativa à aparência física. Dos numerosos resultados obtidos pelo estudo, observou-se que a autoestima relativa à aparência correlacionou de modo estável e elevado com autoestima global, ao passo que em domínios sociais, as correlações com a autoestima geral aumentaram com a idade, especialmente quanto ao apelo romântico à autoestima (von Soest et al., 2016).

Estudos longitudinais que acompanham a autoestima tanto geral como em domínios são importantes ao proverem um panorama mais claro da valorização de si em etapas da vida em que o desenvolvimento da personalidade e da identidade está em momento crítico, como afirmam abordagens clássicas e contemporâneas em psicologia do desenvolvimento e da personalidade (Donnellan, Hill, \& Roberts, 2015; Erikson, 1968/1976). Esse tipo de pesquisa pode ser um bom seguimento para os estudos que utilizaram, em sua maioria, a RSES para fins de validá-la para diversos idiomas.

\section{Considerações Finais}

A avaliação da autoestima provoca questionamentos como: É traço ou estado? Produto do desenvolvimento ou um processo? Fenômeno fundamentalmente psicológico ou sociológico? As respostas não são unânimes. Essa realidade difusa dificulta a elaboração de um instrumento que contemple todas ou boa parte dessas questões. Talvez por esta razão há tantos instrumentos que avaliem autoestima em contextos tão específicos (Mruk, 2013). A própria Rosenberg, que elaborou uma medida unidimensional para avaliação do construto, argumentou que há autoestima global e autoestima relacionada a algum domínio ou situação específica (Rosenberg, Schooler, Schoenbach, \& Rosenberg, 1995).

A autoestima é um construto valorizado em pesquisa. Contudo, não há evidências suficientes que apoiem a crença de que aumentar a autoestima garante a melhoria da qualidade de vida de alguém ou a resolução de algum problema social. Inclusive, algumas manifestações da elevada autoestima são consequência de comportamentos defensivos, narcisistas ou arrogantes. No máximo, 
a autoestima elevada é um indicador de saúde mental, bem-estar e adequação social de uma pessoa (Hewitt, 2009). Não deve, portanto, ser um objetivo almejável per se. Vale a pena, e muito, promover o funcionamento humano ótimo por meio de pesquisa e intervenção que fomente aspectos, como autoeficácia, otimismo, esperança, autocompaixão etc.

A autoestima contingente pode ser uma alternativa interessante para a avaliação da representação de si associada a determinados domínios, como no exemplo dos pacientes a serem submetidos a cirurgias que envolvem o corpo, como a bariátrica, a plástica e os transplantes de órgãos e tecidos. Como geralmente os indivíduos atribuem valor a domínios específicos de suas vidas, estes contribuem para a manutenção da autoestima (James, 1890/1950). Por isso, enquanto em algumas pessoas a autoestima está ligada à aparência, em outras está associada ao desempenho laboral. Assim, existe uma diferença da contingência a ser satisfeita para que se obtenham maiores níveis de autoestima (Crocker \& Wolfe, 2001) e haverá maior esforço nas áreas que julgam relevantes por meio da autoavaliação (Maricutoiu, Macsinga, Ruso, Virga, \& Sava, 2012).

Este trabalho possui limitações. Dois estudos foram conduzidos para averiguar o uso de escalas de autoestima nas publicações. No Estudo 1, foram escolhidos quatro periódicos, limitando, portanto, o escopo de busca e impedindo generalizaçóes para o contexto de todas as revistas nacionais da área da Psicologia. Ademais, não foi analisado o uso de escalas de autoestima em publicações de outras áreas, o que pode ser realizado em pesquisa futura. Já quanto às publicações estrangeiras, no Estudo 2, focalizou-se nas palavras do título que contivessem a expressão "self-esteem scale", para viabilizar uma amostra de tamanho razoável e que de fato contivesse estudos dedicados ao uso da escala. Dessa forma, a limitação está na delimitação da menção da expressão de busca exclusivamente no título do trabalho. Considerado o corpo do resumo, centenas de outras publicações resultariam na busca, material para estudos vindouros.

É de fundamental importância que as pesquisas utilizem instrumentos atualizados quanto a suas propriedades psicométricas, como no caso da RSES por meio dos trabalhos de Hutz e Zanon (2011) e de Hutz et al. (2014). Procedimentos necessários para a correta adaptação cultural e condução de estudos de validade estão descritos em Hutz, Bandeira e Trentini (2015), bem como nas orientações fornecidas pela International Test Commission (ITC) (cf. www.intestcom.org). No presente trabalho, destaca-se que, no mínimo, é preciso mencionar como foi realizada a tradução, adaptação cultural e demais procedimentos de evidências de validade, características básicas da amostra e o coeficiente de consistência interna do instrumento. Pode-se, também, citar o texto que contém as informações psicométricas sobre a escala e sua versão utilizada.

Outro aspecto que deve ser salientado relaciona-se de modo indireto aos resultados dos dois estudos relatados no presente trabalho. Trata-se da publicação de artigos que utilizaram instrumentos adaptados sem informar os procedimentos realizados para a adaptação, nem dados psicométricos básicos. Tendo sido publicados, a reflexão recai sobre o exame realizado pelos pareceristas dos periódicos sobre os textos, bem como sobre o acompanhamento conduzido pelos editores. Nem tudo precisa ser detalhado em um manuscrito, mas há dados que são fundamentais e que interferem diretamente sobre os resultados relatados e discutidos na publicação. Em se tratando de autoestima, isto é, da forma como a pessoa valoriza a si, uma informação mal medida pode gerar resultados inesperados ou errôneos. É preciso que pareceristas e editores, portanto, atentem mais sobre esses aspectos ao avaliarem manuscritos submetidos para publicação.

\section{Referências $^{2}$}

Assis, S., Avanci, J., Silva, C., Malaquias, J., Santos, N., \& Oliveira, R. (2003). A representação social do ser adolescente: Um passo decisivo na promoção da saúde. Ciência \& Saúde Coletiva, 8(3), 669-679. doi: 10.1590/S1413-81232003000300002

Avanci, J., Assis, S., Santos, N., \& Oliveira, R. (2007). Adaptação transcultural de escala de autoestima para adolescentes. Psicologia: Reflexão e Crítica, 20(3), 397-405. doi: 10.1590/S0102-79722007000300007

Baumeister, R., Smart, L., \& Boden, J. (1996). Relation of threatened egotism to violence and aggression: The dark side of self-esteem. Psychological Review, 103(1), 5-33. doi: 10.1037/0033-295X.103.1.5

Brown, J., \& Marshall, M. (2006). The three faces of self-esteem. In M. Kernis (Eds.), Self-esteem issues and answers: A sourcebook of current perspectives (pp. 4-9). New York: Psychology Press.

Capelatto, I., Lima, R., Ciasca, S., \& Salgado-Azoni, C. (2014). Cognitive functions, self-esteem and self-concept of children with Attention Deficit and Hyperactivity Disorder. Psicologia: Reflexão e Crítica, 27(2). doi: /10.1590/1678-7153.201427214

Carneiro, A., \& Baptista, M. (2016). Escala de Pensamentos Depressivos (EPD). São Paulo: Hogrefe.

\footnotetext{
${ }^{2}$ As referências com asterisco constam somente na Tabela 1
} 
Coopersmith, S. (1967). The antecedents of self-esteem. San Francisco: Freeman.

Coopersmith, S. (1989). Self-Esteem Inventory. Palo Alto, CA: Consulting Psychologists Press.

*Costa, B. (2014). Investigando a relação entre autoconceito, autoeficácia e autoestima: Construção de evidências a partir da escala de cognições acadêmicas autorreferentes (Dissertação de mestrado). Universidade Federal de Minas Gerais: Belo Horizonte. Recuperado de http://www. bibliotecadigital.ufmg.br/dspace/handle/1843/BUOS-AU3M76

Costa, B., Gomes, C., \& Fleith, D. (2017). Validade da Escala de Cognições Acadêmicas Autorreferentes: Autoconceito, autoeficácia, autoestima e valor. Avaliação Psicológica, 16(1), 87-96. doi: 10.1569/ap.2017.1601.10

Crocker, J., \& Wolfe, C. (2001). Contingencies of self-worth. Psychology Review, 8(3), 593-623. doi: 10.1037/0033-295X.108.3.593

Dini, G., Quaresma, M., \& Ferreira, L. (2004). Adaptação cultural e validação da versão brasileira da Escala de Autoestima de Rosenberg. Revista da Sociedade Brasileira de Cirurgia Plástica, 19(1), 47-52. Recuperado de www.rbcp.org.br/imageBank/PDF/19-01-04pt.pdf

Donnellan, M., Hill, P., \& Roberts, B. (2015). Personality development across the life span: Current findings and future directions. In M. Mikulincer, P. Shaver, M. Cooper \& R. Larsen (Eds.), APA handbook of personality and social psychology (vol. 4, pp. 107-126). Washington, DC: American Psychological Association.

Erikson, E. H. (1976). Identidade, juventude e crise (2 ed.). Rio de Janeiro: Zahar. (Original publicado em 1968).

García, F., \& Musitu, G. (2014). Manual Autoconcepto Forma 5. Madrid: TEA Ediciones.

Gobitta, M., \& Guzzo, R. (2002). Estudo inicial do Inventário de Autoestima (SEI) - Forma A. Psicologia: Reflexão e Crítica, 15(1), 143-150. doi: 10.1590/S0102-79722002000100016

Harter, S. (1982). The Perceived Competence Scale for children. Child Development, 53(1), 87-97. doi: 10.2307/1129640

Harter, S. (1985). The self-perception profile for children: Revision of the Perceived Competence Scale for Children. Denver, CO: University of Denver.

Harter, S. (1986). Processes underlying the construction, maintenance, and enhancement of the self-concept in children. In J. Suls \& A. Greenwald (Eds.), Psychological perspectives on the self (vol. 3, pp. 137-181). Hillsdale, NJ: Erlbaum.

Hewitt, J. (2009). Self-esteem. Em S. Lopez (Ed.), Encyclopedia of positive psychology (vol. 2, pp. 880-886). Malden, MA: Wiley- Blackwell.

Hutz, C. S. (2000). Adaptação da escala de autoestima de Rosenberg. Manuscrito não publicado. Universidade Federal do Rio Grande do Sul: Porto Alegre, RS.

Hutz, C. S., Bandeira, D., \& Trentini, C. (Eds.) (2015). Psicometria. Porto Alegre: Grupo A.

Hutz, C. S., Midgett, A., Pacico, J., Bastianello, M., \& Zanon, C. (2014). The relationship of hope, optimism, self-esteem, subjective wellbeing, and personality in Brazilians and Americans. Psychology, 5(6), 514-522. doi: 10.4236/psych.2014.56061

Hutz, C. S., \& Zanon, C. (2011). Revisão da adaptação, validação e normatização da Escala de Autoestima de Rosenberg. Avaliação Psicológica, 10(1), 41-49. Recuperado de http://pepsic.bvsalud.org/pdf/avp/v10n1/v10n1a05.pdf

Hutz, C. S., Zanon, C., \& Vazquez, A. (2014). Escala de Autoestima de Rosenberg. In C. S. Hutz (Eds.), Avaliação em psicologia positiva (pp. 85-94). Porto Alegre: Artmed.

James, W. (1950). The principles of psychology. New York: Dover. (Original publicado em 1890).

Judge, T., Erez, A., Bono, J., \& Thoresen, C. (2003). The Core Self-Evaluations Scale (CSES): Development of a measure. Personnel Psychology, 56(2), 303-331. doi: 10.1111/j.1744-6570.2003.tb00152.x

Maricutoiu, L. P., Macsinga, I., Rusu, S., Virga, D., \& Sava, F. A. (2012). Adaptation and validation of the contingencies of self-worth scale on a Romanian student sample. Cognition, Brain, Behavior: An interdisciplinary Journal, XVI(16), 121-138. doi: 10.15303/rjeap.2016. si1.a73

Marsh, H., Craven, R., \& Martin, A. (2013). What is the nature of self-esteem? Unidimensional e multidimensional perspectives. In M. Kernis (Eds.), Self-esteem issues and answers: A sourcebook of current perspectives (pp. 16-25). New York: Psychology Press.

Mruk, C. (2013). Self-esteem and positive psycology: Research, theory, and practice (4a ed.). New York: Springer.

Pasin, E., Paiva, M., \& Lannes, D. (2012). Associação entre fatores psicológicos e relacionais e o rendimento escolar no ensino fundamental. Avaliação Psicológica, 11(2), 275-286. Recuperado de http://pepsic.bvsalud.org/scielo.php?script=sci_arttext\&pid=S167704712012000200013\&lng $=\mathrm{pt} \& \operatorname{tlng}=\mathrm{pt}$

Reppold, C., Mayer, J., Almeida, L., \& Hutz, C. S. (2012). Avaliação da resiliência: Controvérsia em torno do uso das escalas. Psicologia: Reflexão e Crítica, 25(2), 248-255. doi: 10.1590/S0102-79722012000200006

Rosenberg, M. (1965). Society and the adolescent self-image. Princeton, NJ: Princeton University.

*Rosenberg, M. (1979). Conceiving the self. New York: Basic Books.

*Rosenberg, M. (1985). Self-concept and psychological well-being in adolescence. In R. Leahy (Org.), The development of the self (pp. 205246). Orlando, FL: Academic Press.

*Rosenberg, M. (1986). Conceiving the self. Malabar, FL: Krieger Publishing.

*Rosenberg, M. (1989). Society and the adolescent self-image (edição revisada). Middletown, CT: Wesleyan University.

Rosenberg, M., Schooler, C., Schoenbach, C., \& Rosenberg, F. (1995). Global self-esteem and specific self-esteem: Different concepts and different outcomes. American Sociological Review, 60(1), 141-156. doi: 10.2307/2096350

Seligman, M. (1995). The optimistic child: A proven program to safeguard children against depression and build lifelong resilience. New York: Harper Collins.

Souza, L. K., \& Hutz, C. S. (2016). Self-compassion in relation to self-esteem, self-efficacy and demographical aspects. Paideia, 26(64), 181188. doi: 10.1590/1982-43272664201604

Snyder, C., \& Lopez, S. (2002). Handbook of positive psychology. New York: Oxford University Press.

von Soest, T., Wichstrom, L., \& Kvalem, I. (2016). The development of global and domain-specific self-esteem from age 13 to 31 . Journal of Personality and Social Psychology, 110(4), 592-608. http://dx.doi.org/10.1037/pspp0000060 
Zanon, C., Bastianello, M., Pacico, J., \& Hutz, C. S. (2013). Desenvolvimento e validação de uma escala de afetos positivos e negativos. Psico-USF, 18(2), 193-201. doi: 10.1590/S1413-82712013000200003

\section{Sobre os autores}

Daniela Bergesch D’Incao Marrone é psicóloga, especialista em psicologia clínica, mestre e doutoranda em Psicologia do Desenvolvimento pela Universidade Federal do Rio Grande do Sul. Bolsista de Doutorado CNPq.

Luciana Karine de Souza é psicóloga, com mestrado e doutorado pela Universidade Federal do Rio Grande do Sul (UFRGS). Atualmente é professora adjunta na UFRGS, docente-permanente no PPG-Psicologia (UFRGS) e coordenadora do "Et Alia: Laboratório de Desenvolvimento Humano e Relações Interpessoais" (UFRGS-CNPq).

Claudio Simon Hutz é psicólogo, possui mestrado e doutorado pela University of Iowa (USA). Atualmente é professor titular da Universidade Federal do Rio Grande do Sul e coordenador do Laboratório de Mensuração do PPG Psicologia da UFRGS. 